\title{
Coupled Heat, Moisture and Salt Transport in Civil Engineering Structures Performed on Parallel Computers
}

\author{
J. Kruis, T. Krejci, M. Sejnoha, J. Nemecek \\ Department of Mechanics, Faculty of Civil Engineering \\ Czech Technical University in Prague \\ Thakurova 7, 16629 Prague, Czech Republic \\ jk@cml.fsv.cvut.cz, krejci@cml.fsv.cvut.cz \\ sejnom@cml.fsv.cvut.cz, jiri.nemecek@fsv.cvut.cz
}

Many civil engineering structures are composed of heterogeneous and porous materials with complex inner microstructures. An analysis of heat and moisture transfer in a very complicated pore space of such building materials has become an indispensable part of the design of new buildings or reconstructions of existing structures.

Very often, the moisture transport is accompanied with the transport of chemical species such as different kinds of salts originating either from the composition of the material itself or, more frequently, from outer sources. Deicing chemical or salty waters are typical sources of the salinity that infiltrates into the interior of the structural materials. The salts can further act on internal damage of the material caused e.g. by crystallization in pores or depassivation of steel bars in the case of reinforced concrete structures when the tensile strength of the material is exceeded. Such damages can be significant and the serviceability life of the whole structure is thus significantly reduced.

The general computational framework for the coupled heat, moisture and salt transport used for the solution of large scale problems in civil engineering will be covered in the presentation. The solution strategy for solving the system of partial differential equations with finite elements will be shown. The numerical complexity will be treated with the aid of parallel calculation on distributed computers.

The high degree of heterogeneity and complicated pore space crossing all the scales in building materials is very often treated by multi-scale models that separate the scale into distinct levels and decrease the computational complexity of the problem. Also in this presentation, a two-scale model for a composite structure will be introduced. The upper (macro-scale) level of the model describes the whole structure while the meso-scale level describes all necessary details in the material composition. For this task, representative unit cells are used for the description. The unit cell calculation leads to the assessment of homogenized material parameters that are sent as an input to the macro-scale level calculation. After the spatial discretization of the problem, the typical number of degrees of freedom (DOFs) in the finite element model is hundreds of thousands for the macro-scale level. But, hundreds or thousands of DOFs are used in unit cells on the meso-scale level. It means, a problem with thousands of DOFs is solved a hundred thousand times. Moreover, a time integration method has to be used on the macro-scale level since the heat and moisture transfer is described as a non-stationary problem. It is evident, the presented methodology is computationally very demanding and it is suitable for parallel calculation which will be covered in the presentation.

The two-scale model of coupled heat and moisture transfer will be demonstrated on the analysis of a historical Charles bridge in Prague which was built in 14th century from stone bricks and is now after the reconstruction. The Kunzel's model is used and several statistically equivalent unit cells for the meso-scale level are generated from in-situ measurements. The analysis was performed on a parallel computer and a very good speedup was obtained. The temperature and moisture distribution obtained from the analysis were in a very good agreement with the measured values. The second example is devoted to the coupled heat, water and water vapor transfer in a reactor vessel of a nuclear power plant with the aid of the presented computational framework.

\section{Acknowledgement}

Financial support of the Czech Science Foundation (project No. 16-11879S) is gratefully acknowledged. 